\title{
Desenhos de Crianças Submetidas a Cirurgias Eletivas*
}

\author{
Camilla Volpato Broering** @ \& Maria Aparecida Crepaldi (D \\ Universidade Federal de Santa Catarina, Florianópolis, SC, Brasil
}

\begin{abstract}
RESUMO - O presente artigo tem por objetivo investigar as memórias que as crianças têm sobre a cirurgia a qual foram submetidas. Participaram desta pesquisa 80 crianças com idade entre 6 e 12 anos, internadas num hospital infantil para a realização de cirurgia eletiva de pequeno porte, divididas em quatro grupos de intervenção. Foram utilizados desenhos como forma de instrumento de coleta de dados. A pesquisa ocorreu em duas etapas: na primeira, cada grupo recebeu a sua preparação no hospital, e na segunda, cerca de quinze dias após a cirurgia. Pôde-se constatar, após uma análise dos desenhos, que todos os grupos retrataram a condição de hospitalização e cirurgia, e trouxeram detalhes sobre os aspectos relacionados a esta temática.
\end{abstract}

PALAVRAS-CHAVE: memórias pós-cirúrgicas, psicologia pediátrica, cirurgia na infância

\section{Children's Drawings Submitted to Elective Surgeries}

\begin{abstract}
This article investigates the memories that children have about the surgery that they had undergone. Participants of this study were 80 children aged between 6 and 12 years, hospitalized in a children's hospital for the performance of small elective surgeries, divided into four intervention groups. Drawings were used as a form of data collection instrument, this being a widespread practice with children. The study took place in two stages: first, each group received their preparation in the hospital then they were asked to produce drawings, approximately 15 days after the surgery, in their homes. After an analysis of the drawings, it was noted that all groups portrayed the condition of hospitalization and surgery and presented details about the aspects related to this issue.
\end{abstract}

KEYWORDS: postsurgical memories, pediatric psychology, surgery in childhood

\section{INTRODUÇÃO}

Este artigo apresenta os efeitos da preparação psicológica pré-cirúrgica sobre os desenhos das crianças submetidas a cirurgias eletivas, bem como discutir as suas limitações. Assim, refere-se às cirurgias eletivas em geral, por serem mais pesquisadas, e fornecerem subsídios para outras pesquisas sobre memórias infantis e seu impacto nas memórias infantis.

Como se trata de uma pesquisa realizada na casa da criança, portanto, após a cirurgia, tem-se que os desenhos feitos por elas referiram-se às memórias que tiveram sobre o processo de hospitalização e cirurgia. Em se falando de memórias, sabe-se que, até recentemente, recém-nascidos que necessitassem de cirurgias não eram sequer anestesiados ou, tampouco, recebiam analgesia pós-operatória. Acreditava-se na ausência da dor, porém, felizmente, as justificativas anteriormente apresentadas, tais como imaturidade do sistema nervoso central, ausência de memória para a dor e a falta de pesquisas acerca do tema não mais se fundamentam, segundo Anand el al. (2000). Hoje existem inúmeros trabalhos provando que até mesmo o recém-nascido pré-termo (RNPT) já possui o substrato neurofisiológico desenvolvido para captar o estímulo doloroso. Dessa forma, o tema em questão ganhou importância, adotando-se em alguns hospitais a inclusão da dor como quinto sinal vital, até mesmo na área pediátrica.

Sobre este tema, foram encontrados poucos estudos, o que fica evidente na revisão de literatura realizada por

\footnotetext{
* Artigo proveniente da Tese de Doutorado da primeira autora sob supervisão da segunda autora.

** E-mail: millavolbro@hotmail.com

- Submetido: 10/10/2016; Revisado: 15/07/2019; Aceito: 27/04/2020.
} 
Broering (2014). Desse modo, devido à incidência de cirurgias na infância e as reações das crianças hospitalizadas frente às mesmas, torna-se conspícuo avaliar as memórias que as crianças têm de suas cirurgias como forma de investigação e atuação profissional, pautada na realidade explicada pelas próprias crianças que já passaram por essa situação. Assim, pode-se proporcionar um melhor entendimento sobre a cirurgia, que favoreça a comunicação dos adultos com a criança doente.

Os procedimentos próprios da internação hospitalar envolvem desde a administração de um medicamento até a realização de cirurgias, que incluem imunizações, punções venosas e biópsias, procedimentos que costumam gerar dor e ansiedade e acabam por adquirir caráter aversivo (Uman et al., 2008). Desse modo, torna-se necessário e eficaz o uso da preparação psicológica com o objetivo de reduzir o estresse e a ansiedade frente ao desconhecido.

A lembrança da dor e da situação traumática é trazida à tona pela existência de memória do evento traumático. A habilidade de recordar ou reconhecer experiências anteriores é uma das definições de memória. Os tipos de memória mais estudados em humanos são a implícita (inconsciente, a qual não pode ser descrita em palavras) e a explícita (consciente e que pode ser descrita). A implícita é aquela sobre a qual os indivíduos conseguem demonstrar conhecimento ou habilidade, mas não podem resgatar explicitamente as informações. Em crianças com menos de um ano, a memória para eventos dolorosos tem como forma de expressão as mudanças no comportamento. É comum percebê-las mais vigilantes na presença de profissionais de saúde usando um jaleco branco. Já a memória explícita é aquela com a qual os indivíduos conseguem encontrar determinado objeto e indicar a consciência de qual é o correto (Sapolnik et al., 2007).

Desse modo, torna-se importante que as crianças submetidas a cirurgias eletivas passem por algum tipo de preparação psicológica pré-cirúrgica, com vias de que tenham memórias salutares sobre seus procedimentos cirúrgicos.

\section{Preparação Psicológica Pré-cirúrgica}

Os benefícios da preparação de crianças para cirurgia e demais procedimentos invasivos já eram objeto de estudo desde a década de 60 , bem como a importância da participação dos pais na hospitalização como fatores importantes no combate ao estresse e consequências nocivas da internação, além de ser medida fundamental para proteger a criança de danos em seu desenvolvimento (Crepaldi \& Hackbarth, 2002).

Momentos pré-cirúrgicos podem ser vivenciados pelo paciente com medo frente ao desconhecido e isso mostra-se como a principal causa da insegurança e da ansiedade do paciente pré-cirúrgico (Fighera \& Viero, 2005).
Le Roy et al. (2003) recomendam que se deva fazer uma avaliação acurada da criança e de suas condições psicossociais e enumeram alguns fatores importantes a serem avaliados junto à família, tais como: o nível de desenvolvimento da criança e seu estilo de enfrentamento; a compreensão da criança e da família sobre sua condição médica e sobre o procedimento médico a ser realizado; experiência prévia de hospitalização e, particularmente, de situações adversas; sintomas emocionais, cognitivos e físicos; medos em geral e de procedimentos específicos; composição familiar, incluindo fatores linguísticos, culturais e religiosos; o método mais apropriado para lhes transmitir as informações (verbal, visual, escrita e sensorial); outros estressores familiares, como os financeiros, sociais, e outros eventuais problemas de saúde; o modo segundo o qual os familiares tomam decisões.

A preparação psicológica pré-cirúrgica da criança e dos pais é fundamental, pois lhes possibilita certo grau de controle sobre o desconhecido que a situação cirúrgica representa, pois, em geral, esta é sentida e percebida como um momento de vulnerabilidade e risco. A informação é uma intervenção que atenua a ansiedade e o estresse, permitindo ao paciente e ao familiar esclarecimento sobre os procedimentos, o que possibilita maior controle sobre a situação estressora (Crepaldi et al., 2006; Magalhães et al., 2010; Ribeiro \& Pinto, 2009). Gaíva e Silva (2014) incentivam que os profissionais dialoguem com os pais sobre os eventos significativos da saúde da criança, envolvam a família, forneçam informações sobre os cuidados e os problemas de saúde, bem como as incluam nas intervenções.

Para a redução da ansiedade, medo e problemas suscitados pelo desconhecimento da situação, gerando mais segurança e conforto aos pequenos pacientes, é fundamental e necessário informações simples e curtas sobre os procedimentos e o centro cirúrgico contribuem (Faleiros et al., 2002; Miskowski, 2008; Rossit \& Fávere, 2011).

Por outro lado, Motta e Enumo (2004) pontuam que o brincar é utilizado no hospital tanto com crianças como com os profissionais para lidarem com as adversidades da hospitalização. Uma pesquisa realizada por Paladino et al. (2014) com 30 crianças submetidas a cirurgias ortopédicas, de adenoidectomia e postectomia, destacou a preparação para cirurgia por meio do brincar como eficaz, visto que os comportamentos apresentados pelas crianças antes da cirurgia demonstraram interesse pela brincadeira, participando ativamente ao fazer perguntas, repetir a brincadeira e manusear os brinquedos hospitalares com desenvoltura.

Magalhães et al. (2010) também realizou um estudo, avaliando três crianças com idade entre 9 e 14 anos. A avaliação com aplicação de escalas foi realizada em dois momentos que antecediam a realização da cirurgia, pré e pós-preparo psicológico. Os resultados do prépreparo evidenciaram ocorrência de medo, ansiedade e preocupações. No preparo psicológico, os participantes 
puderam experienciar de forma lúdica o procedimento cirúrgico, além de tirar dúvidas, fazer questionamentos e desmistificar crenças relacionadas ao procedimento médico. Na reavaliação pós-preparo, houve diminuição dos sintomas que apareceram anteriormente.

Silva e Zakir (2011) estudaram 20 pacientes em situação de preparo pré-cirúrgico e avaliaram os efeitos da intervenção por nível de cortisol sanguíneo, Inventário de Ansiedade de Beck, comportamento na Unidade de Terapia Intensiva e evolução clínica. Foram encontradas diferenças estatísticas significativas entre o grupo experimental e o grupo controle em relação ao comportamento menos agitado na Unidade de Terapia Intensiva, nível de cortisol sanguíneo antes da cirurgia, decréscimo no grau de ansiedade e evolução clínica. A intervenção parece ter produzido efeito positivo no comportamento e na evolução clínica do paciente.

Desse modo, os efeitos da hospitalização para a realização de uma cirurgia podem ser minimizados com a inclusão de estratégias de preparação das crianças antes de sua consecução, o que deve incluir a presença e preparação concomitante dos pais. Por meio da forma como a criança vive e é influenciada por essa experiência, considera-se importante avaliar também como as crianças lembram dessas experiências ocorridas e como essas memórias sobre a hospitalização e cirurgia podem afetar o seu desenvolvimento.

\section{Memórias Pós-Cirúrgicas em Crianças}

Em pesquisa realizada por Broering (2014), foram encontrados poucos estudos com crianças referentes a memórias infantis sobre cirurgias. No geral, o estudo de Sapolnik et al. (2007) aponta que existe memória de dor em crianças e que pode causar sequelas, caso passem por novas experiências dolorosas. Mas, nesse caso, tem-se que são referidas memórias dolorosas.

De acordo com Sapolnik et al. (2007), avaliar a memória da dor em Pediatria é algo subjetivo, porém viável. O tema em questão tem despertado interesse com o intuito de criar novos métodos de avaliação e o acompanhamento psicológico mostrou-se fundamental.

No que ser refere às cirurgias, mesmo que os contínuos avanços nas práticas cirúrgicas e anestésicas, devidas ao desenvolvimento científico e tecnológico, às políticas de redução de custos e aumento de eficácia do tratamento médico, tenham resultado no declínio do tempo médio de internação hospitalar (Gilmartin \& Wright, 2007; Mitchell, 2000; Rankinen et al., 2007), a preparação psicológica pré-cirúrgica dos indivíduos ainda é um tema recorrente em psicologia da saúde e em outras ciências (Broering \& Crepaldi, 2013; Rankinen et al., 2007).
A pesquisa de Broering (2008), cujo objetivo foi averiguar os efeitos de dois programas de preparação psicológica no estresse e na ansiedade de crianças submetidas a cirurgias eletivas, levantou outras questões de interesse e, em virtude dessas questões, teve-se o interesse em aperfeiçoar a pesquisa anteriormente realizada com a inclusão de outras variáveis.

Alguns pacientes submetidos a procedimentos cirúrgicos apontam motivos para o aumento da ansiedade: a) percepção antecipada de dor e desconforto; b) espera passiva pelo início do procedimento; c) separação da família e sensação de abandono; d) possível perda, de autonomia; e) medo da morte, de sequelas, do procedimento de anestesia e do risco de alta prematura; e f) a cirurgia em si (Bellani, 2008; Berg, et al., 2006; Garbee \& Gentry, 2001; Gilmartin \& Wright, 2008; Marcolino et al., 2007). Tais motivos podem elevar a pressão sanguínea e causar sangramentos, que podem prejudicar a recuperação pós-cirúrgica (Ribeiro et al., 2002).

Uma preparação pode vir a aumentar a probabilidade do desenvolvimento de estratégias mais eficientes de enfrentamento do procedimento cirúrgico, colaboração com a equipe médica, melhora do processo de comunicação, diminuição dos níveis de estresse e ansiedade e, consequentemente, otimização do tempo de recuperação cirúrgica e alta hospitalar, como meios de evitar danos ao desenvolvimento e proporcionar memórias mais saudáveis.

Uma cirurgia geralmente acarreta algum impacto sobre o bem-estar físico, social e emocional do paciente, afastamento da rede de apoio familiar e, por assim dizer, tem-se que a análise funcional da preparação psicológica de pacientes para cirurgia é um tema legítimo de pesquisa também pelos benefícios da sua utilização. Porém, poucos foram os estudos encontrados que mencionem o resultado dessa preparação após a realização de um procedimento cirúrgico e, especialmente, quais as lembranças que as crianças têm desse período e de que forma estas lembranças podem influenciar seu desenvolvimento. Saber sobres essas memórias traria resultados possivelmente positivos, que ajudariam profissionais de saúde a incrementar a atuação dos porfissionais como forma de subiscidiar suas práticas, pautadas no relato das crianças sobre o que gostaram e o que foi de difícil manejo para elas. Assim, pode-se promover uma melhor compreensão sobre a cirurgia, por parte da criança, de modo que esse processo seja mais tranquilo e saudável, com uma comunicação efetiva entre profissionais e pacientes.

Assim, este artigo apresenta os efeitos da preparação psicológica pré-cirúrgica sobre os desenhos das crianças submetidas a cirurgias eletivas em situação pós-cirúrgica, bem como discute as limitações que se apresentam para a pesquisa nesta área da psicologia e da saúde da criança. 


\section{MÉTODO}

\section{Participantes}

Participaram desta pesquisa 80 crianças ( 40 meninos e 40 meninas) com idade variando entre 6 e 12 anos, internadas num hospital infantil para a realização de cirurgia eletiva de pequeno porte. As intervenções cirúrgicas foram: hérnias inguinal e umbilical, amigdalectomia, adenoidectomia e postectomia.

\section{Instrumentos}

A utilização de desenhos como forma de avaliação de crianças é muito difundida na prática psicológica. Quando utilizado em pesquisa para coleta de dados, pressupõe-se que sejam considerados forma, traços, escolha e uso das cores como elementos que possam contemplar a análise de conteúdo veiculado. Acrescentar-se-ia a esses aspectos o conteúdo verbalizado sobre o desenho, através de histórias contadas ou comentários realizados pela criança enquanto envolvida com a atividade de desenhar (Fávero \& Salim, 1995).

\section{Procedimentos}

Com a autorização para a realização do estudo, a pesquisadora recebia o mapa cirúrgico do hospital e fazia o primeiro contato com as famílias e crianças, quando era explicado sobre os passos da pesquisa. Com os Termos de Consentimento assinados pela instituição, pela criança e pelo responsável, teve-se acesso aos dados de identificação e endereço da criança.

\section{Rapport e Preparação Psicológica Pré-Cirúrgica}

Nesse momento, as crianças fizeram seu primeiro contato com a pesquisadora e foram convidadas a participar da pesquisa, visto que foi explicado o estudo tanto para a criança como para o acompanhante. Mediante a demonstração de interesse de ambos em participar, foi pedido ao responsável e à criança para assinar o Termo de Consentimento Livre e Esclarecido. As crianças foram divididas em quatro grupos, alternando-se os grupos à medida que as cirurgias foram acontecendo.

\section{Constituição dos Grupos de Pesquisa}

O primeiro grupo foi composto por 20 crianças, as quais receberam informações verbais, sobre o tipo de cirurgia que realizariam, todas as etapas citadas anteriormente. As informações consistiram em contar para a criança as etapas pelas quais ela passaria em sua cirurgia, incluindo anestesia (cheirinho), tubo de respiração, curativo e retorno ao quarto. Esse procedimento ocorreu em, no máximo, 30 minutos.

O segundo grupo, também com 20 crianças, as quais foram submetidas a um programa de preparação précirúrgica. Por meio de materiais hospitalares, a criança manuseava e os utilizava, para torná-la mais próxima da realidade a que seria submetida. Desse modo, as informações foram dadas às crianças à medida que ela brincava com o boneco. Esse programa também foi aplicado após todas as etapas previamente citadas, com duração de 30 minutos.

No terceiro grupo, também com 20 crianças, elas assistiram a um vídeo explicativo sobre a cirurgia e receberam informações verbais.

Por sua vez, o quarto grupo constituiu-se de 20 crianças que não receberam nenhum tipo de preparação psicológica pré-cirúrgica.

Todas as atividades citadas foram realizadas no dia anterior à cirurgia.

\section{Coleta de Dados}

Após 15 dias da realização da cirurgia, deu-se a segunda etapa da coleta de dados, que foi realizada na residência de cada criança. A técnica de coleta de dados utilizada foi o Desenho para evocar memórias. Nesse momento, pediu-se para a criança confeccionar um desenho, após ouvir a instrução: "Desenhe sobre o que você lembra sobre sua hospitalização. Preste bastante atenção, porque depois vou pedir para que você me conte sobre o que desenhou". Assim que a criança terminava o desenho, perguntava-se sobre o que a criança havia desenhado e acrescentava-se, quando necessário, perguntas simples para esclarecer os comentários da criança.

\section{Considerações Éticas}

Os procedimentos éticos foram seguidos e obteve-se a aprovação no comitê de ética pela Universidade Federal de Santa Catarina, sob número 120.114. Outros cuidados foram a assinatura dos Termos de Consentimento, tanto por parte dos responsáveis como por parte das crianças. Justificou-se o fato de ter um grupo de crianças que não recebeu preparação para cirurgia pelo fato de o hospital não possuir nenhum programa pré-cirúrgico prévio.

\section{Análise de Dados}

A análise dos desenhos foi baseada nos critérios descritos por Fávero e Salim (1995), que consideram as seguintes características: cor escolhida; característica do traço utilizado; tamanho; detalhes presentes no desenho; e 
verbalizações durante o desenho. Os desenhos passaram por uma análise desenvolvimentista, realizada através de duas microanálises: análise dos estádios de desenvolvimento das competências e análise dos estádios de expressão artística e de desenvolvimento cognitivo (Menezes, 2010).

Os desenhos analisados tiveram por objetivo verificar as memórias das crianças sobre a cirurgia e foram realizados cerca de 15 dias após a realização da cirurgia, na residência da criança, em horário previamente marcado pelos pesquisadores.

Analisou-se os desenhos com o intuito de perceber a capacidade das crianças para desenhar, forma do traçado, conteúdo e harmonia do desenho. Porém, foram observados o gênero e a idade das mesmas, para que não ocorressem discrepâncias no processo de análise, visto que as crianças tinham idades variando entre 6 e 12 anos.

\section{RESULTADOS}

O quadro a seguir demonstra a análise dos aspectos representativos nos desenhos dos diferentes grupos, de acordo com o conteúdo e o traçado (Figura 1).

\section{Análise dos Desenhos do G I}

Pode-se observar que no G1 houve presença de traçados correspondentes à insegurança e ansiedade, revelado através de desenhos com rabiscos e sombreamentos. Observou-se a presença de alguns desenhos com traçado sem detalhes, próximo à fase mais precoce do desenvolvimento da criança.

Com relação ao conteúdo, observou-se que a maior parte dos desenhos se referiu ao quarto e ao centro cirúrgico antes da ocorrência da cirurgia. Ademais, desenhos referentes à chegada no hospital, fachada do hospital, cama do quarto, roupa do hospital e trajeto para o centro cirúrgico também ocorreram. Em menor proporção, ocorreu um desenho da família, fora do contexto hospitalar, relacionado à preparação que, em perspectiva, destacou a presença da psicóloga no quarto, seguido "da criança indo ao centro cirúrgico", "a criança no centro cirúrgico" e "a criança em casa após a cirurgia".

Houve um desenho fazendo referência a um baralho que foi levado pelo pai da criança com o intuito de passarem o tempo; outro desenho referiu-se ao banho. Desenhos sobre os equipamentos cirúrgicos e hospitalares, usados pelas enfermeiras para verificar os sinais vitais no quarto antes e depois da cirurgia, também apareceram. Outro caso foi de um desenho em que a criança se desenhou como um bebê no leito do hospital, o que denota regredir sua atual idade, que era de 9 anos.

No geral, destaca-se que esses desenhos do G1 trouxeram informações e conteúdo sobre a situação hospitalar momentos antes da cirurgia. Observou-se também que os desenhos com figuras humanas eram sem expressão de sentimentos. Outro fator importante foi a presença de rabiscos em metade dos desenhos, o que pode sugerir insegurança.

\section{Análise dos Desenhos do G2}

No G2, houve presença de traçados correspondentes à insegurança e ansiedade, revelados através de desenhos com rabiscos e sombreamentos. Alguns desenhos denotaram indícios de medo de separação, visto que aparecia a mãe de um lado da folha, e a criança do outro, separados pelo centro cirúrgico.

No que se refere ao conteúdo, verificou-se uma variedade de detalhes e situações apresentadas nos desenhos. A maioria fez menção ao Centro Cirúrgico e aos profissionais médicos e enfermeiras. Em vários desenhos, notou-se a criança indo encontrar o médico no centro cirúrgico e, em outros, apareceu a sala de observação sem a criança, ressaltando-se que essas crianças permaneceram nessa sala antes de ter sido liberada a internação, visto que o hospital estava sem leito disponível quando chegaram. Nesse grupo, encontrou-se a maior variedade de situações hospitalares: mãe no centro cirúrgico com a criança; sala de recuperação, com e sem a criança; quarto do hospital com muitos detalhes (pia, televisor, janelas, ar condicionado, banheiro, chuveiro, lâmpada, suporte de soro, cadeiras dos acompanhantes, cobertor). Em alguns desenhos, a criança estava presente no quarto e em outros a criança estava ausente e o desenho não constava de figura humana.

Foram situações representadas nos desenhos: o hospital em tamanho grande e a casa, ao lado, em tamanho consideravelmente menor do que o do hospital; quarto do hospital distante da casa da criança; a mãe no quarto esperando a hora do filho ir para o centro cirúrgico;

\begin{tabular}{|c|c|c|c|c|}
\hline Grupos & G1 & G2 & G3 & G4 \\
\hline Traçado & $\begin{array}{c}\text { Insegurança } \\
\text { Ansiedade }\end{array}$ & $\begin{array}{c}\text { Insegurança } \\
\text { Ansiedade e de separação }\end{array}$ & Insegurança & $\begin{array}{c}\text { Insegurança } \\
\text { Ansiedade } \\
\text { Medo }\end{array}$ \\
\hline Conteúdo & $\begin{array}{c}\text { Quarto e centro cirúrgico } \\
\text { antes da cirurgia }\end{array}$ & $\begin{array}{c}\text { Centro cirúrgico e } \\
\text { profissionais }\end{array}$ & $\begin{array}{c}\text { Centro cirúrgico e quarto } \\
\text { após a cirurgia }\end{array}$ & $\begin{array}{c}\text { Hospital e casa antes da } \\
\text { cirurgia }\end{array}$ \\
\hline
\end{tabular}

Figura 1. Aspectos dos desenhos nos diferentes grupos 
mãe e filha chorando antes de ir para o centro cirúrgico; desenhos dos instrumentos cirúrgicos distribuídos pela folha (termômetro, estetoscópio, tesoura, pijama hospitalar, maca e soro, instrumentos estes utilizados na preparação); um desenho da hérnia que seria operada; ambulância em frente ao hospital; e um desenho do contorno da mão da criança e ao redor dela, a cama, cadeiras, hospital e portas.

Pode-se ressaltar que pouco menos da metade dos desenhos do G2 apresentou riqueza de detalhes e em apenas um desenho observou-se que o contexto retratado se referiu à situação pós-cirúrgica, pois todos os demais se remetiam ao momento pré-cirúrgico. É evidente, assim como no G1, que esses desenhos trouxeram informações e conteúdo sobre a situação hospitalar nos momentos anteriores à cirurgia, pois apenas uma criança desenhou conteúdo relacionado ao pós-cirúrgico. Os desenhos com figuras humanas apresentaram-se sem expressão de sentimentos, seja felicidade ou expressão de outros sentimentos e, além disso, ressalta-se que muitas crianças se desenharam dormindo. Pouco menos da metade dos desenhos apresentou rabiscos, o que pode sugerir, assim como ocorrido no G1, a presença de insegurança.

\section{Análise dos Desenhos do G3}

Com relação ao traçado dos desenhos do G3, observou-se que houve presença de alguns traçados correspondentes à insegurança, revelado através de desenhos com rabiscos. Porém, não se evidenciaram indicativos de ansiedade ou medo, conforme relatos das crianças. A maioria dos desenhos teve boa qualidade gráfica, sem a presença de rabiscos, com contornos definidos e precisos.

Com relação ao conteúdo, observou-se que a maior parte dos desenhos se referiu ao centro cirúrgico e quarto do hospital após a realização da cirurgia, com muitos detalhes, tais como cama, poltrona do cuidador, pia, televisor, ar condicionado, janelas e lâmpadas. Pôde-se observar que, em um desenho, apareceu o sangue na situação pós-cirúrgica; no entanto, a criança que confeccionou esse desenho sofreu um acidente de carro ao sair do hospital no trajeto para casa e, em virtude disso, teve que retornar ao hospital devido a um sangramento. Uma grande parte das crianças desse grupo fez menção às atividades de preparação realizadas pelos pesquisadores. $\mathrm{O}$ centro cirúrgico também foi desenhado algumas vezes com riqueza de detalhes, tais como vidros que separam o centro cirúrgico da sala de recuperação, luzes, imagens das paredes do centro cirúrgico e equipamentos.

Foram observados os desenhos em perspectiva, os quais mostravam o quarto hospitalar, o centro cirúrgico e, por fim, a casa. Nesses casos, tanto teve desenhos que denotavam que as crianças estavam felizes como aqueles nos quais não havia figura humana. Outros, não trouxeram a casa, mas sim o centro cirúrgico seguido da sala de recuperação, enfatizando que o desenho se remetia ao momento após a cirurgia e as crianças estavam felizes por isso. Houve desenhos que enfatizaram a mãe e a criança saindo do hospital felizes. Outras situações trazidas nos desenhos foram: a mãe esperando a criança voltar do centro cirúrgico com expressão de felicidade, e a criança chegando ao quarto chorando pelo efeito da anestesia, como fora mencionado por ela mesma, e a comparação entre o quarto da criança e o quarto do hospital, também com expressão de felicidade.

Portanto, o G3 foi caracterizado por apresentar riqueza de detalhes, independente da faixa etária, e a maioria dos desenhos mencionou o momento pós-cirúrgico, a volta para casa e a preparação psicológica realizada. Foram poucos os desenhos sem figura humana e quase todos apresentaram expressão de felicidade.

\section{Análise dos Desenhos do G4}

A partir da análise do traçado do desenho, os desenhos do G4, na sua maioria, mostraram a presença de traçados correspondentes à insegurança e ansiedade, revelados por meio de desenhos com rabiscos e sombreamentos. Também teve alguns desenhos com indícios de medo do procedimento a que as crianças seriam submetidas.

No conteúdo, em geral o hospital e o centro cirúrgico foram desenhados com detalhes, tais como: luzes, camas, poltronas, pia, equipamentos cirúrgicos, televisor, ar condicionado, portas e janelas. Em alguns desenhos, deu-se ênfase à porta do hospital em tamanho grande, ao lado da porta do quarto em tamanho consideravelmente menor. Além disso, desenhos referentes aos equipamentos cirúrgicos, a criança no quarto antes de ir para o centro cirúrgico, a criança no quarto em tamanho menor do que realmente é e a criança indo para o centro cirúrgico também ocorreram. Outros desenhos trouxeram menção à presença de sangue e de comida no quarto. Em menor proporção, notou-se desenhos que se referiam a duas situações, tais como a casa da criança seguida do hospital e o quarto do hospital seguido do centro cirúrgico. Uma criança desenhou o pesquisador aplicando os testes. Observou-se que a maior parte dos desenhos desse grupo referiu-se ao hospital e à casa antes do procedimento cirúrgico.

Vale ressaltar que, em alguns desenhos, mais de uma das situações citadas acima ocorreram simultaneamente, isto é, o desenho que mostrava a criança no quarto antes de ir para o centro cirúrgico também apresentou a criança em tamanho menor, ou o desenho que mostrava o quarto em detalhes elucidava também a situação na casa antes de ir para o hospital.

Desse modo, os desenhos do G4, em geral, tiveram uso de cores em sua maioria, com apresentação de rabiscos e sombreamento e menção significativa aos momentos antes da cirurgia. Nesse grupo, não apareceram desenhos sem figura humana e o conteúdo em todo o grupo disse respeito a aspectos do hospital e do centro cirúrgico. 


\section{DISCUSSÃO}

Constatou-se, após uma análise geral dos desenhos, que todos os grupos retrataram a condição de hospitalização e cirurgia e trouxeram detalhes sobre os aspectos relacionados a essa temática.

Houve diferenças no traçado do desenho no que diz respeito a cores, harmonia e preenchimentos de acordo com a idade das crianças, porém essa diferença não ocorreu devido ao tipo de preparação recebida, visto que, no geral, em todos os grupos, os desenhos de crianças da mesma faixa etária se assemelharam. Em todos os grupos e no que tange a idade, as crianças mais velhas tiveram uma tendência a habilidades mais refinadas para representar o conteúdo dos desenhos e as crianças mais jovens fizeram desenhos mais coloridos. Porém, as crianças mais velhas pareceram não se dedicar tanto ao desenho, pois são desenhos rápidos, simples e com poucas cores. Nas crianças mais jovens, houve uma tendência aos rabiscos, pouca definição de traçados e ausência de contornos, no entanto, o processo de confecção do desenho foi mais demorado e elas o fizeram com mais dedicação e empenho. Em decorrência do desenvolvimento infantil, sabe-se que, quanto mais velha é a criança, mais fidedignas são as informações obtidas sobre uma dada situação. Entretanto, Von Baeyer (2004) pontuou que até mesmo bebês mostram evidências de memória declarativa, mesmo que num curto espaço temporal.

Os grupos G1, G2, e G4 trouxeram conteúdo referente ao momento que antecedia a cirurgia, seja indo para o hospital, o próprio hospital, o quarto do hospital ou o centro cirúrgico. Já o G3 trouxe desenhos relacionados à situação pós-cirúrgica, seja ainda no centro cirúrgico, no quarto, ou na situação de voltar para casa. Foram observados desenhos em perspectiva, os quais trouxeram as situações desde a ida ao hospital, a cirurgia e a volta para casa.

Outra diferença entre os grupos referiu-se à quantidade de crianças que fizeram desenhos sobre a preparação psicológica recebida no G3. No G4, apenas uma criança fez essa referência. Vale lembrar que esse grupo não recebeu nenhuma preparação, então a menção foi à aplicação dos testes. No G1, também teve apenas um desenho que fez menção à preparação, mostrando-a num desenho em perspectiva, ou seja, que trouxe também outras situações. Apesar de na faixa etária dos 6 aos 8 anos a tendência ter sido de que os desenhos fossem mais rabiscados, no G3, mesmo nessa faixa de idade, isso não ocorreu. Crianças com mais idade elaboram mais suas respostas e as crianças mais jovens fantasiam mais, o que não implica em inverdades, mas em trazer mais detalhes sobre um determinado assunto ou evento. Não houve diferenças no que concerne a gênero e tipo de cirurgia.

No que diz respeito à expressão de sentimentos nos grupos, verificou-se que no $\mathrm{G} 3$ a maior parte dos desenhos apresentava crianças sorrindo, demonstrando felicidade, conquanto, isto não foi observado nos demais grupos, nos quais os desenhos com figura humana eram sem expressão de sentimentos. O choro apareceu no G3 em um desenho em que a criança mencionou a preparação, na qual foi falado que a anestesia poderia causar choro na volta da criança para o quarto; foi assim que a criança desenhou o choro, como efeito da anestesia. Nos outros grupos, o choro apareceu como característica de medo frente à cirurgia.

Em relação a presença de sangue nos desenhos, percebeu-se que apareceu apenas no G4. Os resultados deste artigo coadunam com os resultados encontrados por Sapolnik et al. (2007), quando afirma que crianças têm memória de dor, pois, nesse artigo, as crianças relataram a dor como principal reação após a cirurgia. Em consequência disso, a experiência cirúrgica pode trazer marcas em futuras experiências de crianças que tiveram uma experiência não tão boa. Assim, percebe-se a importância de conhecer os pensamentos e percepções das crianças sobre suas experiências cirúrgicas, de modo que futuras intervenções baseiem-se nisto.

Observou-se também que, em relação à qualidade do traçado, independente da faixa etária, em todos os grupos, com exceção do G3, metade ou mais da metade dos desenhos apresentaram rabiscos e sombreamentos, o que pode sugerir ansiedade e insegurança. Além disso, o medo apareceu apenas nos grupos $\mathrm{G} 2$ e G4, mas com entonações diferentes, no G2 apareceu como medo de separação da mãe e, no G4, como medo da cirurgia.

Pôde-se notar que no G1 e no G4 ocorreram desenhos em que a criança se desenhou como um bebê no quarto do hospital e este tipo de desenho não ocorreu nos G2 e G3. Ademais, pode-se afirmar que os desenhos do G3 apresentaram melhor qualidade de traçado, menos rabiscos, desenhos com contorno, harmoniosos e riqueza de detalhes em todas as faixas etárias. Nos outros grupos, houve maior discrepância entre as faixas etárias e a riqueza de detalhes ficou mais destacada a partir dos 9 anos de idade.

A partir do exposto, verificou-se que em todos os grupos as crianças demonstraram em seus desenhos que têm memórias sobre o que ocorreu no hospital, seja da preparação, do hospital, do centro cirúrgico, do quarto ou dos colegas de quarto. O que difere é o enfoque que é dado à situação da cirurgia, a maneira como lembram e como expressam isso através do desenho.

As três técnicas utilizadas aparecem na literatura como efetivas na preparação de crianças (Bess d'Alcantara, 2008; Moix, 1996; Watson \& Visram, 2003; Yamada \& Bevilacqua, 2005), o que coaduna com os resultados desta pesquisa, nos três programas preparatórios, os quais basearam-se na informação, seja ela narrada, assistida, ou vivenciada. Tesser e Prebianchi (2013), em seu estudo de revisão bibliográfica, também tiveram seus achados corroborando as técnicas utilizadas neste estudo, porém não compararam as técnicas ou verificaram o tamanho do efeito delas. 


\section{CONSIDERAÇÕES FINAIS}

Os resultados deste estudo ratificam a importância da utilização do desenho como recurso de investigação de processos psicológicos com crianças. Utilizam-se desenhos no cotidiano das crianças, que se utilizam dos mesmos em suas brincadeiras para expressarem suas emoções e significações de experiências vividas. Ainda que o desenho, na condição de método de reconhecimento, possa evocar um volume menor de informações ou estimular a fantasia da criança, como técnica ainda necessita ser melhor averiguada. O potencial do uso do desenho como método não deve ser generalizado para pesquisas de outras naturezas.

No tangente às memórias e aos programas de preparação psicológica pré-cirúrgica, houve diferenças entre o tipo de preparação realizado e o conteúdo das memórias. De acordo com as entrevistas e desenhos, destaca-se que o G3 trouxe memórias referentes à preparação realizada bem como aos testes aplicados e referiu-se às situações relacionadas ao pós-cirúrgico e volta para casa, diferente dos outros grupos que trouxeram menção à situação hospitalar, quarto de hospital e centro cirúrgico. Ademais, o G4 teve memórias relacionadas a choro, expressões de tristeza nos desenhos, sangue e dúvidas.
Pelo exposto, observa-se que há diferenças nas memórias de crianças submetidas a cirurgias eletivas, de acordo com o tipo de preparação e em relação ao grupo de controle. O programa de preparação psicológica pré-cirúrgica que propiciou à criança a visualização do vídeo explicativo trouxe maiores benefícios em relação às memórias posteriores de crianças submetidas a cirurgias eletivas.

Em relação às memórias pós-cirúrgicas, percebeu-se que os grupos retrataram a condição de hospitalização e cirurgia, e trouxeram detalhes sobre os aspectos relacionados a essa temática. No entanto, numa comparação entre os grupos de preparação psicológica, verificou-se que os desenhos do G3 apresentaram melhor qualidade de traçado em todas as faixas etárias, bem como desenhos relacionados à situação pós-cirúrgica, seja ainda no centro cirúrgico, no quarto, ou na situação de voltar para casa. Esses achados diferiram dos outros grupos que trouxeram conteúdo referente ao momento que antecedia a cirurgia, seja indo para o hospital, o próprio hospital ou o centro cirúrgico. Concluiu-se que o G3 parece apresentar resultados mais positivos em relação aos demais grupos, embora isso não desmereça os demais tipos de preparação.

\section{REFERÊNCIAS}

Anand, K.J.S., McGrath P., Schechter N., \& Levetown M. (2000). A dor na infância. Nestlé Nutricion Services.

Bellani, M. L. (2008). Psychological aspects in day-case surgery. International Journal of Surgery, 6 (1), 44-46. http://dx.doi. org/10.1016/j.ijsu.2008.12.019

Berg, A., Fleischer, S., Koller, M., \& Neubert, T. R. (2006). Preoperative information for ICU patients to reduce anxiety during and after the ICU-stay: Protocol of a randomized controlled trial. Biological Medical Central Nursing, 5 (1), 4-11. http://dx.doi.org/10.1186/1472-6955-5-4

Bess d'Alcantara, E. (2008). Criança hospitalizada: O impacto do ambiente hospitalar no seu equilíbrio emocional. Revista Virtual de Psicologia Hospitalar e da Saúde, 3 (6), 38-55.

Broering, C. V. (2014). Efeitos da preparação psicológica pré-cirúrgica em crianças a serem submetidas a cirurgias eletivas e suas memórias. [Tese de Doutorado em Psicologia]. Universidade Federal de Santa Catarina.

Broering, C.V. \& Crepaldi, M.A. (2013). O estudo das memórias pós-cirúrgicas: importância e limitações. Vitalle, 25 (2), 53-61.

Broering, C. V. (2008). Efeitos da preparação psicológica précirúrgica em crianças a serem submetidas a cirurgias eletivas. [Dissertação de Mestrado em Psicologia]. Universidade Federal de Santa Catarina.

Crepaldi, M.A. \& Hackbarth, I.D. (2002). Aspectos psicológicos de crianças hospitalizadas em situação pré-cirúrgica. Temas em Psicologia da SBP, 10 (2), 99-112

Crepaldi, M.A., Rabuske, M.M., \& Gabarra, L.M. (2006). Modalidades de atuação do psicólogo em psicologia pediátrica. In M. A. Crepaldi, B. M. Linhares, G.B. Perosa, (Org.). Temas em Psicologia Pediátrica. (pp. 13-55). Casa do Psicólogo.

Faleiros, F., Sadala, M. L. A., \& Rocha, E. M. (2002). Relacionamento terapêutico com criança no período perioperatório: utilização do brinquedo e da dramatização. Revista da Escola de
Enfermagem da USP, 36(1), 58-65. http://dx.doi.org/10.1590/ S0080-62342002000100009

Fávero, M.H. \& Salim, C.M.R. (1995). A relação entre os conceitos de saúde, doença e morte: Utilização do desenho na coleta de dados. Psicologia: Teoria e Pesquisa, 11, 181-191.

Fighera, J., \& Viero, E.V. (2005). Vivências do paciente com relação ao procedimento cirúrgico: fantasias e sentimentos mais presentes. Revista Brasileira de Psicologia Hospitalar, $8(2), 51-63$.

Gaíva, M.A.M. \& Silva, F.B. (2014). Caderneta de Saúde da Criança: Revisão Integrativa. Revista de Enfermagem da UFPE, 8 (3), 742-749

Garbee, D. D., \& Gentry, J. A. (2001). Coping with the stress of surgery. Association of PeriOperative Registered Nurses Journal, 73 (5), 946-951. http://dx.doi.org/10.1016/S00012092(06)61746-8

Gilmartin, J., \& Wright, K. (2007). The nurse's role in day surgery: A literature review. International Nursing Review, 54 (2), 183190. http:qqdx.doi.orgq10.1111qj.1466-7657.2007.00528.x

Gilmartin, J., \& Wright, K. (2008). Day surgery: Patients' felt abandoned during the preoperative wait. Journal of Clinical Nursing, 17 (18), 2418-2425. http://dx.doi.org/10.1111/j.13652702.2008.02374.x

Le Roy, S., Elixson, E. M., O’Brien, P., Tong, E., Turpin, S., \& Uzark, K. (2003). Recommendations for preparing children and adolescents for invasive cardiac procederes. Circulation: Journal of the american heart association, 108, 2550-2564.

Magalhães, F. M., Gusmam, D. P. P., \& Grecca, K. R. R. (2010). Preparo psicológico em pacientes submetidos a cirurgia cardíaca pediátrica. Revista Brasileira Terapia Cognitiva 6(2), 144-166.

Marcolino, J. A. M., Suzuki, F. M., Alli, L. A. C., Gozzani, J. L., \& Mathias, L. A. S. T. (2007). Medida da ansiedade e da depressão 
em pacientes no pré-operatório: estudo comparativo. Revista Brasileira de Anestesiologia, 57 (2),157-166. http://dx.doi. org/10.1590/s0034-70942007000200004

Menezes, M. (2010). A criança e sua rede familiar: significações do processo de hospitalização. [Tese de Doutorado em Psicologia] Universidade Federal de Santa Catarina, Florianópolis, S.C.

Miskowski, E. M. P. (2008). Intervenção psicológica em crianças a serem submetidas a cirurgia através do brinquedo terapêutico diretivo. [Monografia de Graduação em Psicologia]. Universidade Federal de Sergipe.

Mitchell, M. (2000). Anxiety management: a distinct nursing role in day surgery. Ambulatory Surgery, 8(3), 119-127. http://dx.doi. org/10.1016/S0966-6532(99)00061-X

Moix, J. (1996). Preparación psicológica para la cirurgia en pediatria. Archivos de pediatria, 47 (4), 211-217.

Motta, A.B., \& Enumo, S.R.F. (2004). Brincar no hospital: estratégia de enfrentamento da hospitalização infantil. Psicologia em Estudo, 9 (1), 19-28. http://dx.doi.org/10.1590/S141373722004000100004

Paladino, C.M., Carvalho, R., \& Almeida, F.A. (2014). Brinquedo terapêutico no preparo para a cirurgia: Comportamentos de pré-escolares no período transoperatório. Revista da Escola de Enfermagem da USP, 48 (3), 423-429. doi: http://dx.doi. org/10.1590/S0080-623420140000300006

Rankinen, S., Salanterä, S., Heikkinen, K., Johansson, K., Kaljonen, A., Virtanen, H., \& Leino-Kilpi, H. (2007). Expectations and received knowledge by surgical patients. International Journal for Quality in Health Care, 19 (2), 113-119. http://dx.doi org/10.1093/intqhe/mzl075

Ribeiro, C. R., \& Pinto Junior, A. A. (2009). A representação social da criança hospitalizada: um estudo por meio do procedimento de desenho-estória com tema. Revista da Sociedade Brasileira de Psicologia Hospitalar, 12(1), 31-56.

Ribeiro, R. M., Tavano, L. D. A., \& Neme, C. M. B. (2002). Intervenções psicológicas nos períodos pré e pós-operatório com pacientes submetidos a cirurgia de enxerto ósseo. Estudos de Psicologia (Campinas), 19 (3), 67-76. https://doi. org/10.1590/S0103-166X2002000300007

Rossit, R. A. S. \& Fávere, D. C. (2011). Influência de atividades pedagógicas sobre o comportamento de crianças hospitalizadas e seus acompanhantes. Revista Brasileira de Terapia Comportamental e Cognitiva, 23(3), 52-67.

Sapolnik, R., Almeida, P., \& Souza, M. (2007). Memória da Dor em Crianças Oncológicas. Pediatria Moderna, 45 (4), 146.154.

Silva, M.E.M., \& Zakir, N.S. (2011). Controle instrucional e relaxamento como preparo psicológico pré-cirúrgico para portadores de cardiopatia. Estudos de Psicologia (Campinas), 28 (3), 371-379. http://dx.doi.org/10.1590/S0103166X2011000300009

Tesser, N.L., \& Prebianchi, H.B. (2013, setembro). Preparação psicológica de pacientes adultos e infantis para cirurgia: Uma revisão. [Anais] XVIII Encontro de Iniciação Científica. Campinas, SP, Brasil.

Uman, L. S., Chambers, C. T., McGrath, P. J., \& Kisely, S. (2008). A systematic review of randomized controlled trials examining psychological interventions for needle-related procedural pain and distress in children and adolescents: An abbreviated Cochrane Review. Journal of Pediatric Psychology. 33(8),842854. http://dx.doi.org/10.1093/jpepsy/jsn031

Von Baeyer, C. (2004). Children's memory for pain: Overview and implications for practice. J. Pain, 5, 241-249. http://dx.doi. org/10.1016/j.jpain.2004.05.001

Watson, A.T. \& Visram, A. (2003). Children's preoperative anxiety and postoperative behaviour. Paediatric Anaesthesia, 13, 188-204.

Yamada, M.O. \& Bevilacqua, M.C. (2005). O trabalho do psicólogo no programa de implante coclear do Hospital de Reabilitação de Anomalias Craniofaciais. Estudos de Psicologia (Campinas), 22 (3), 255-262. 